\title{
Analysis of Complexity and Power Consumption in DSP-Based Optical Modulation Formats
}

\author{
J. L. Wei, Q. Cheng, R. V. Penty, and I. H. White \\ Centre for Photonic Systems, Electrical Engineering Division, Department of Engineering, University of Cambridge, $9 \mathrm{~J} J$ Thomson Avenue, \\ Cambridge CB3 OFA, UK. E-mail: jw748@cam.ac.uk \\ D. G. Cunningham \\ Avago Technologies, Framlingham Technology Centre, Station Road, Framlingham, Suffolk, IP13 9EZ, UK.
}

\begin{abstract}
Analysis shows that $400 \mathrm{GbE}$ links using DSP-enabled 5×80Gb/s PAM-4, 4×100Gb/s hybrid CAP-16/QAM-16 and 4×100Gb/s QAM-16-OFDM have relative complexities of 1:5.4:5.7 and consume power of $0.71,0.67$ and 0.75 times that of $16 \times 25 \mathrm{~Gb} / \mathrm{s}$ NRZ benchmark links. OCIS codes: (060.2330) Fiber optics communications; (060.4080) Modulation;
\end{abstract}

\section{Introduction}

Internet traffic continues to grow exponentially fuelled by bandwidth-hungry applications such as high definition TV, video-on-demand, online gaming/conferencing/healthcare, virtual private networks, and cloud computing etc. To fulfil the bandwidth demand, optical fibre data links have been widely adopted for high speed short-haul interconnectivity. Intensity modulation and direct detection (IMDD) systems are preferred in data links due to their cost-effectiveness. Although digital signal processing (DSP) has gained strong momentum mainly in long haul transmissions where digital coherent receivers are used [1], the use of DSP in short-haul applications has also attracted significant attention due to the continuous advances in CMOS technology [2-4]. In this respect, the complexity of the DSP solution has become a critical factor in determining the system cost of short reach communication systems. Moreover, power dissipation has gained more importance in the ICT sector as a result of concerns about global warming. Thus energy-efficiency is also an important criterion for technology evolution [5].

Ethernet has been the dominant technology for short reach optical interconnects [6]. In March 2013, the IEEE 802.3 started the $400 \mathrm{~Gb} / \mathrm{s}$ Ethernet Study Group and most likely that multi-lane solutions will be adopted due to component bandwidth limitations [7]. It is possible that 1st generation $400 \mathrm{GbE}$ might be based on 8 lanes with 50 $\mathrm{Gb} / \mathrm{s}$ rate lane rate over 2 fiber pairs, and the 2 nd generation may adopt 4 lanes each operating at $100 \mathrm{~Gb} / \mathrm{s}$ based on only 1 fiber pair [7]. Recent research efforts have shown a number of DSP-based demonstrations of various advanced modulation formats appropriate for such applications. There have been $50 \mathrm{~Gb} / \mathrm{s}$ per polarization PAM-4 signal transmission over $100 \mathrm{~m}$ SMF link [8], $102 \mathrm{~Gb} / \mathrm{s}$ multiband CAP signal transmission over $15 \mathrm{~km} \mathrm{SMF} \mathrm{[2],}$ $112 \mathrm{~Gb} / \mathrm{s}$ quadrature amplitude modulation-16 (QAM-16) over a 4 km SMF link [3], and $100 \mathrm{~Gb} / \mathrm{s}$ optical OFDM system with $10 \mathrm{~km}$ SMF transmission [4]. All these demonstrations are based on IMDD and components having a bandwidth of $\sim 25 \mathrm{GHz}$. This paper aims to provide the first known comprehensive review of the above mentioned modulation schemes on DSP complexity and power dissipation for 400 Gigabit Ethernet scenarios.

\section{System Architecture}

Fig. 1 depicts the system architectures for a single lane $50 \mathrm{~Gb} / \mathrm{s}$ PAM-4, $100 \mathrm{~Gb} / \mathrm{s}$ hybrid CAP-16/QAM-16, and $100 \mathrm{~Gb} / \mathrm{s}$ 16-QAM-OFDM. Components having bandwidth $\sim 25 \mathrm{GHz}$ and directly modulated DFB lasers (DMLs) are considered based on parameters detailed in [9], which indicates only CAP and OFDM can support single lane $100 \mathrm{~Gb} / \mathrm{s}$ transmission while PAM-4 cannot. Therefore, 4 (8) transceivers are considered for $4 \times 100 \mathrm{~Gb} / \mathrm{s}$ CAP/QAM and OFDM $(8 \times 50 \mathrm{~Gb} / \mathrm{s}$ PAM-4) to analyze the overall DSP complexity and power dissipation. For PAM-4, the encoded PAM symbols first passed through a pre-equalizer prior to conversion to analogue pulses via a DAC. After electrical to optical (E/O) conversion based on a DML, the optical PAM signal propagates through a fibre link and is converted into an electrical signal by a photo-diode (PD) followed by signal processing in the receiver that is the inverse of that in the transmitter. In practise, the equalizers in PAM-4 transceivers are used to mitigate the ISI caused by the RF circuit/path. For hybrid CAP-16/QAM-16, it generates a QAM like signal by combining two multi-level signals using two electrical filters whose impulse responses form an orthogonal Hilbert pair [10]. At the receiver, a modified QAM receiver is used to eliminate the crosstalk between I and Q channels that a CAP receiver otherwise has $[11,12]$. Such a hybrid CAP/QAM system offers not only improved tolerance to timing jitter but also increased system power margin compared with a conventional CAP receiver [11, 12]. For OFDM, the generation of an electrical OFDM signal in the OFDM transmitter is performed by an OFDM modem, 
whose major operations include data modulation format mapping using QAM-16 constellations, power loading, inverse fast Fourier transform (IFFT), cyclic prefix insertion, OFDM symbol serialization and DAC. The generated electrical OFDM signal is then converted into an optical signal. On the receiver side, the optical OFDM signal is converted back into an electrical signal. The received electrical OFDM signal is then digitized and processed by the receiver OFDM modem with an inverse procedure compared to that adopted in the OFDM transmitter.

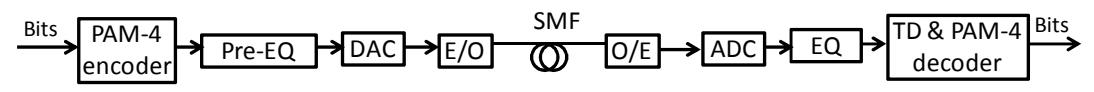

(a) PAM-4

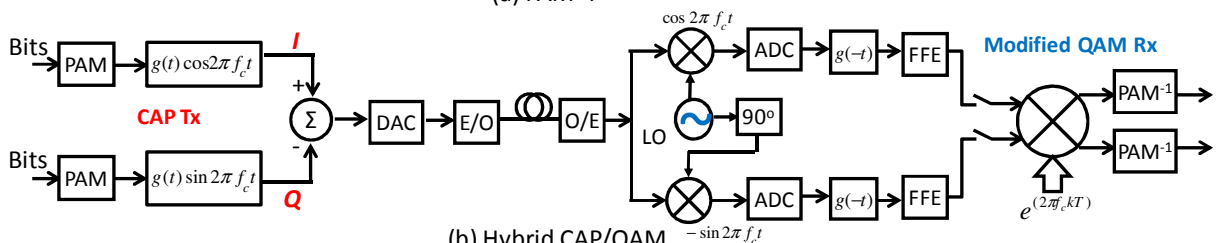

(b) Hybrid CAP/QAM

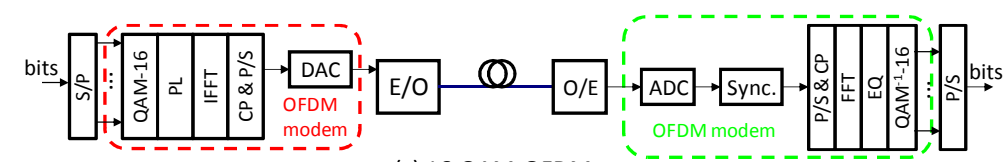

(c) 16-QAM-OFDM

EQ: equalization E/O: electrical to optical convertor TD: threshold device DAC/ADC: digital to analogue/analogue to digital conversion

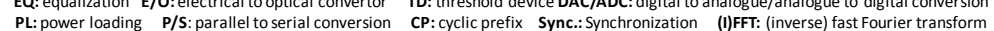

Fig. 1 System architecture a single lane (a) PAM-6, (b) hybrid CAP-16/QAM-16 and (c) QAM-16-OFDM.

\section{Results and Discussions}

The DSP complexity is evaluated as the number of basic real-valued arithmetic operations required. The DSP blocks considered only contain the transmitter components prior to the DAC and the receiver components after the ADC, as shown in Fig. 1. Taking PAM-4 as an example, the transceiver blocks include the PAM-4 encoder/decoder, equalizers and threshold device (TD). PAM-4 is the simplest architecture and thus is considered to be a suitable reference system. The arithmetic operations of the PAM- 4 transceiver include 4 comparisons per symbol in the worst case for the PAM-4 encoder or decoder based on a look-up table, 3 comparisons in the worst case for the TD. A simple 2 tap T space FFE based pre-equalization or receiver equalizer needs 2 multiplications and 1 addition. In total, 17 operations per symbol are required for a single lane $50 \mathrm{~Gb} / \mathrm{s}$ PAM-4 link. Thus the overall required operations per second for $400 \mathrm{GbE}$ using PAM- 4 is given by $8 \times 17 /$ Ts with Ts being the symbol period. For CAP-16/QAM-16, the transceiver DSP blocks include two PAM-4 encoders/decoders and two 12-tap T/4 spaced shaping filters/matched filters, two 20-tap T/4 space FFE filters, one symbol rotator operating at symbol rate and two TDs. The single lane hybrid CAP-16/QAM-16 transceiver needs 184 operations. For 16-QAM-OFDM, the transceiver involves a number of DSP blocks including a QAM-16 encoder/decoder, power loading, IFFT/FFT, receiver symbol synchronization, FFT and single-tap equalization. Detailed analyses of the complexity of each DSP component of the CAP-16/QAM-16 and 16-QAM-OFDM transceivers are not described here due to space but will be presented in the conference.

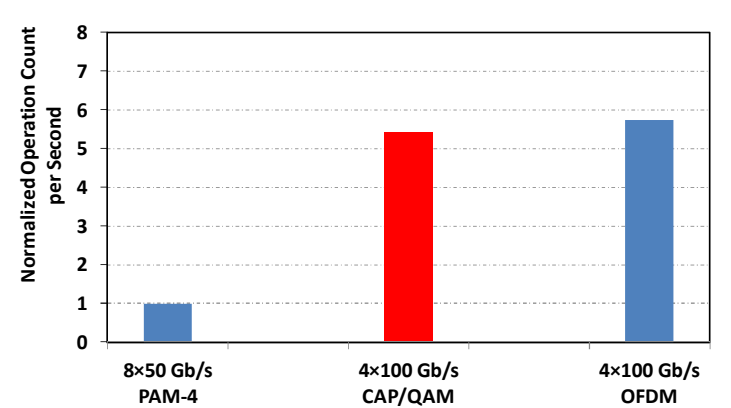

Fig. 2 Normalized arithmetic operation count per second for each scheme.

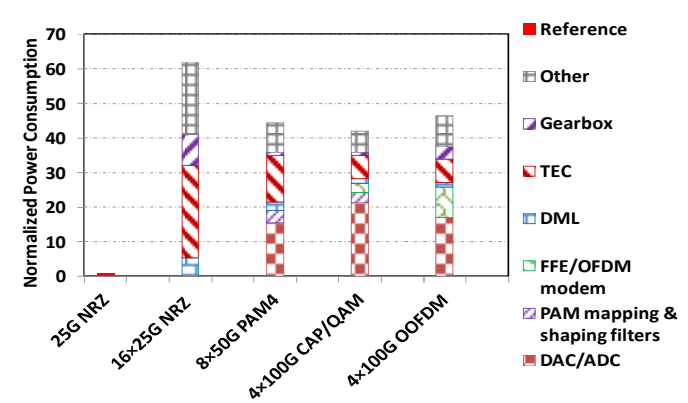

Fig. 3. Normalized transceiver power dissipation for each scheme.

The normalized arithmetic operation count per second for a $400 \mathrm{GbE}$ link using the proposed modulation schemes are summarized in Fig. 2. The total number of operation count is normalized to that of the reference PAM- 
4 system. It shows that PAM-4 needs the least computational effort to support a $400 \mathrm{~Gb} / \mathrm{s}$ overall net bit rate simply because of its relatively simple transceiver configuration, while CAP-16/QAM-16 and QAM-16-OFDM shows about 5.4 and 5.7 times operations relative to that of PAM-4.

The power dissipation of various 400 Gigabit Ethernet links are estimated assuming the use of $65 \mathrm{~nm}$ CMOS technology and a benchmark 100GBASE-LR4 link which adopts 4 parallel DWDM lanes with each lane operating at $25 \mathrm{~Gb} / \mathrm{s}$. The single lane $25 \mathrm{~Gb} / \mathrm{s} \mathrm{NRZ} \mathrm{of} \mathrm{100GBASE-LR4} \mathrm{has} \mathrm{a} \mathrm{transceiver} \mathrm{power} \mathrm{dissipation} \mathrm{of} \mathrm{about} 1$ watt without considering thermoelectric cooler (TEC), which is used to normalize the power consumption of the various $400 \mathrm{GbE}$ modulation format schemes considered here. The transceiver power consumption for each scheme takes into account all transceivers to support $400 \mathrm{~Gb} / \mathrm{s}$ data transmission. A pair of WDM MUXs/DeMUXs is required for each fiber link. Fig. 3 indicates that the benchmark $16 \times 25 \mathrm{~Gb} / \mathrm{s}$ NRZ system consumes the most power compared with the other schemes. This is mainly attributed to the TEC used in each transmitter to stabilize laser wavelength, which typically has a power consumption as high as $1.5 \mathrm{~W}$ [13]. The power dissipations of $8 \times 50 \mathrm{~Gb} / \mathrm{s} \mathrm{PAM}-4$, $4 \times 100 \mathrm{~Gb} / \mathrm{s}$ hybrid CAP/QAM and OFDM are approximately 0.71, 0.67 and 0.75 times that of 16 lane NRZ links, respectively, as a direct result of reduced number of TECs and other components.

It should be noted from Fig. 3 that the DAC/ADCs consume substantial power. Analogue implementations can be adopted for PAM-4 and hybrid CAP/QAM without the need of DAC/ADCs and thus can further improve power efficiency [12]. Alternatively, photonic integration can improve system power efficiency. For example, four lasers of an optical module based on the above-mentioned modulation formats can share one TEC instead of four via integration. This leads to about $75 \%$ reduction of TEC power consumption shown in Fig. 3. If a Course WDM wavelength plan was used, instead of DWDM, it may be possible to use uncooled DML's which could halve the total power dissipation of all schemes.

\section{Conclusions}

The first known comprehensive review on 400 Gigabit Ethernet using advanced DSP-enabled modulation formats has been performed with quantitative analysis of DSP complexity and transceiver power dissipation. Results have shown that $8 \times 50 \mathrm{~Gb} / \mathrm{s}$ PAM-4 has the least complexity, while $4 \times 100 \mathrm{~Gb} / \mathrm{s}$ hybrid CAP-16/QAM-16 and QAM-16OFDM have a complexity of 5.4 and 5.7 times to that of PAM-4, respectively. The three schemes consume power of $0.71,0.67$ and 0.75 times that of a $16 \times 25 \mathrm{~Gb} / \mathrm{s}$ NRZ benchmark link.

\section{References}

[1] B. Spinnler, “Equalizer Design and Complexity for Digital Coherent Receivers” IEEE JSTQE, 16, 1180-1192, (2010).

[2] M. I. Olmedo, et al., "Towards 400GBASE 4-lane solution using direct detection of MultiCAP signal in 14 GHz bandwidth per lane," in proceeding of OFC, Paper PDP5C.10, (2013).

[3] J. C. Cartledge, et al., "100 Gb/s Gbit/s using intensity modulation and direct detection,” in proceeding of ECOC, Paper We. 4. C. 3, (2013).

[4] W. Yan, et al., "100 Gb/s optical IM-DD transmission with 10G-class devices enabled by 65 GSamples/s CMOS DAC Core," in proceeding of OFC, Paper OM3H. 1, (2013).

[5] The GreenTouch Consortium, "GreenTouch Roadmap: Strategic Research Areas and Project Portfolio," (2012).

[6] IEEE, IEEE $802.3 \mathrm{ba}-40 \mathrm{~Gb} / \mathrm{s}$ and $100 \mathrm{~Gb} / \mathrm{s}$ Ethernet, 2010. Standard.

[7] IEEE 802.3 Ethernet Working Group, “400 Gigabit Ethernet Call-For-Interest Consensus,” IEEE 802 March 2013 Plenary, Orlando, FL.

[8] R. Rodes, et al., "100 Gb/s single VCSEL data transmission link,", , in proceeding of OFC, Paper PDP5D, (2012).

[9] J. L. Wei, et al., "Performance Studies of 100 Gigabit Ethernet Enabled by Advanced Modulation Formats," IEEE 802.3 Next Generation 40 $\mathrm{Gb} / \mathrm{s}$ and $100 \mathrm{~Gb} / \mathrm{s}$ Optical Ethernet Study Group. Minneapolis, MN, USA, (2012).

[10] J. J. Werner, Tutorial on carrierless AM/PM, ANSI X3T9.5 TP/PMD Working Group, 1992\&1993.

[11] J. L. Wei, et al, "100 Gigabit Ethernet Transmission Enabled by Carrierless Amplitude and Phase Modulation Using QAM Receivers," in proceeding of. OFC, Paper OW4A.5. (2013).

[12] J. L. Wei, et al., "Experimental Demonstration of Optical Data Links using a Hybrid CAP/QAM Modulation Scheme," Opt. Letters, 39, 1402-1405, (2014).

[13] J. J. Lee, et al., "Predication of TEC Power Consumption for Cooled Laser Diode Module," in proceeding of LEOS, Paper WW3, (2004).

Acknowledgments This work was supported by UK EPSRC via the INTERNET project. 